\title{
GESTÃO DO CONHECIMENTO E GESTÃO DA INOVAÇÃO: UM ESTUDO BIBLIOMÉTRICO NA WEB OF SCIENCE (1945-2015)
}

\author{
KNOWLEDGE MANAGEMENT AND MANAGEMENT \\ INNOVATION: A BIBLIOMETRIC STUDY ON WEB OF \\ SCIENCE (1945-2015)
}

\author{
Paulo Fernando Marschner ${ }^{1}$ \\ Lucas Veiga Ávila ${ }^{1}$ \\ Analisa Tiburski Sommer ${ }^{1}$
}

\begin{abstract}
Recebido em: 07/09/2016 Aceito em: 17/11/2016

paulofernandomarschner@hotmail.com
\end{abstract}

Resumo: Este estudo tem como objetivo analisar as características das publicações sobre Knowledge management (Gestão do conhecimento) e Innovation management (Gestão da inovação) na base de dados Web of Science, no período de 1945 a 2015. O trabalho descritivo e quantitativo, de natureza bibliométrica, busca levantar as características da produção acadêmica. Como principal resultado das 372 publicações analisadas constatou-se que os anos com maior publicação foram os de 2008 e 2015, em especial nas seguintes áreas temáticas: Business economics (Economia Empresarial), Operations research management science (Gestão de Operações), Engineering (Engenharias), Computer science (Ciência da Computação), Information science library science (Ciência da informação/biblioteconomia), Social science (Ciências Sociais). Os documentos são 66,6\% proceedings paper, e o principal titulo é o International journal of technology management. Os países com maior número de produção são a China e os Estados Unidos, e o principal idioma é a língua inglesa.

Palavras-chave: Gestão do Conhecimento. Gestão da Inovação. Bibliometria.

Abstract: This study aims to analyze the characteristics of publications about knowledge management and innovation management in the database Web of Science, in the period 1945-2015. The descriptive and quantitative work, bibliometric nature, seeks to raise the characteristics of academic production. The main result of 372 publications analyzed it was found that the more years the publication were 2008 and 2015, in particular in the following areas: Business economics, Operations research management science, Engineering, Computer science, Information science library science. The documents are $66.6 \%$ proceedings paper, and the main title is the International journal of technology management. The countries with the largest number of production are China and the United States, and primary language is English.

Keywords: Knowledge Management. Innovation Management. Bibliometrics.

\footnotetext{
${ }^{1}$ Universidade Estadual do Rio Grande do Sul - UERGS
} 


\section{INTRODUÇÃO}

Nos últimos anos, presencia-se um contexto de grande valorização dos aspectos intangíveis nas organizações, e com isso começa a emergir uma nova forma de pensar e fazer a gestão do conhecimento. Os desafios e exigências decorrentes desse novo ambiente têm requerido das organizações um ambiente cada vez mais propício ao desenvolvimento de inovações.

A necessidade de gerenciar o conhecimento teve maior fortalecimento a partir do rápido avanço da tecnologia da informação e dos processos de reengenharia, que ocasionaram a redução do quadro funcional nas organizações. Com base nisso o surgimento da gestão do conhecimento é uma consequência do ambiente instável das organizações, que são consideradas capazes de acumular e produzir conhecimento para a alavancagem de inovações (BEHR; NASCIMENTO, 2008).

Para Nonaka e Takeuchi (1997) a partir de várias tentativas para priorizar o conhecimento como recurso importante para as organizações, à gestão do conhecimento procura tratar do aproveitamento dos recursos de informação de que as pessoas aplicam na empresa a fim de criar, compartilhar, e comparar recursos de sucesso como referência para a tomada de decisão, ou seja, é um conjunto de práticas que procuram gerenciar as circunstâncias que o conhecimento precisa para prosperar na organização.

Rosário (2011) aponta que boas políticas de gestão conduzem de forma mais fácil às organizações a resultados inovadores, que assim dotadas com os atributos da inovação conseguem encarar o mercado com alguma vantagem competitiva, e de outro lado à inovação que discorreu de tais políticas de gestão apenas é possível graças a essas mesmas políticas.

Dessa forma a gestão da inovação envolve as atividades relativas à captação, ao uso e ao compartilhamento de conhecimentos pela organização. Os sistemas adequados à gestão da inovação podem melhorar a competitividade e capacidade inovadora (OCDE, 2006).

O tema desta pesquisa foi selecionado para o levantamento de informações bibliométrica, com propósito de realizar maior aprofundamento das características das publicações, dentre elas os temas com maiores destaque na última década, como os países e suas singularidades nas pesquisas, instituições de ensino em destaque, pesquisadores com estudos avançados.

Com base no exposto, este estudo teve como objetivo analisar as características das publicações sobre Knowledge management (Gestão do conhecimento) e Innovation management (Gestão da inovação) na base de dados Web of Science, no período de 1945 a 2016. O presente estudo está organizado da seguinte maneira: inicialmente, está apresentada uma contextualização sobre a gestão do conhecimento e gestão da inovação. Em seguida, demonstra-se o método; após apresenta-se a análise e discussão dos resultados e, por fim, as considerações finais.

\section{GESTÃO DO CONHECIMENTO E GESTÃO DA INOVAÇÃO}

A gestão do conhecimento trata da criação, identificação, integração, recuperação, partilha e utilização do conhecimento no âmbito organizacional, é ainda inclinado para a criação e organização dos fluxos de informação com a intenção de gerar, desenvolver e partilhar o conhecimento para 
assim incentivar a troca deste entre as pessoas para que cada uma consiga agregar valor aos processos e produtos da organização (GIUOMAR, 2014).

Na percepção de Nonaka e Takeuchi (1997) a gestão do conhecimento cuida do capital intelectual das organizações com o objetivo do seu crescimento. Ao contrário da informação o conhecimento diz respeito a crenças e compromissos, é um processo humano dinâmico que faz a interação entre a crença pessoal com relação à verdade. Esse processo assume duas dimensões: uma ontológica e outra epistemológica. A ontológica aponta que o conhecimento só é criado por pessoas, o que indica a premissa de que a criação do conhecimento organizacional amplia o conhecimento criado pelos seus colaboradores e incorpora-o como conhecimento da organização. Já a epistemológica aponta que o homem faz uma reflexão sobre o conhecimento produzido por ele próprio e sobre ele mesmo, sua validade prática, seus estágios de desenvolvimento e suas limitações.

Segundo Nonaka e Takeuchi (1997) há dois tipos de conhecimento: o tácito e o explícito. O conhecimento tácito é aquele que é obtido pelos indivíduos e apresenta dificuldades de codificação, ou seja, não se encontra formalizado em meio concretos, é o tipo de conhecimento que o indivíduo forma a partir das experiências em que é, e se expõe, desta forma ele é pessoal e particular de cada um. Já o conhecimento explícito é formal e objetivo, fácil de ser armazenado e transmitido, é o conhecimento que pode ser registrado e codificado em qualquer formato exterior às pessoas. Pode ser representados por textos, revistas, livros e documentos.

Alarcon (2015) e Dávila (2016) ao retomarem em seus estudos a literatura sobre a gestão do conhecimento identificam diversos autores e conceitos (NONAKA; TAKEUCHI, 1997; SVEIBY, 1998; GURTEEN; 1998; DAVENPORT; PRUSAK, 1998; ALAVI; LEIDNER, 2001; SERVIN, 2005; TERRA, 2005; JENNEX; SMOLNIK E CROASDELL, 2009; NORTH; KUMTA, 2014), e assim os organizam sob uma ótica evolutiva, conforme a Quadro 1.

Quadro 1 - Definição de Gestão do Conhecimento por autores da área

\begin{tabular}{|l|l|}
\hline $\begin{array}{l}\text { NONAKA; TAKEUCHI } \\
\text { (1997) }\end{array}$ & $\begin{array}{l}\text { GC é um processo pelo qual as organizações buscam novas formas de } \\
\text { criar e expandir o conhecimento. }\end{array}$ \\
\hline SVEIBY (1998) & $\begin{array}{l}\text { GC é a arte e o processo de gerar valor, a partir dos ativos intangíveis da } \\
\text { organização. }\end{array}$ \\
\hline GURTEEN (1998) & $\begin{array}{l}\text { GC é um conjunto emergente de projetos operacionais e princípios } \\
\text { organizacionais, em processos, estruturas, aplicações e tecnologias que } \\
\text { auxiliam os "trabalhadores do conhecimento" a alavancar a criatividade e } \\
\text { a capacidade de agregar valor ao negócio. }\end{array}$ \\
\hline $\begin{array}{l}\text { DAVENPORT E } \\
\text { PRUSAK (1998) }\end{array}$ & $\begin{array}{l}\text { GC é o conjunto de ações que envolve identificar, gerenciar, capturar e } \\
\text { compartilhar as informações da organização. }\end{array}$ \\
\hline $\begin{array}{l}\text { ALAVI; LEIDNER } \\
\text { (2001) }\end{array}$ & $\begin{array}{l}\text { GC envolve diferentes etapas, interdependentes e relacionados à criação, } \\
\text { captura, armazenamento e compartilhamento do conhecimento sendo um } \\
\text { fenômeno contínuo, complexo e dinâmico. }\end{array}$ \\
\hline
\end{tabular}




\begin{tabular}{|l|l|}
\hline SERVIN (2005) & $\begin{array}{l}\text { GC é realizada através de três componentes básicos: pessoas, processos } \\
\text { e tecnologia. }\end{array}$ \\
\hline TERRA (2005) & $\begin{array}{l}\text { GC passa, pela compreensão das características e demandas do } \\
\text { ambiente competitivo e pelo entendimento das necessidades individuais e } \\
\text { coletivas associados aos processos de criação e aprendizagem. }\end{array}$ \\
\hline BEJARANO (2006) & $\begin{array}{l}\text { GC é definida como um conjunto de métodos para aquisição, atualização, } \\
\text { armazenamento, disponibilização, manutenção da qualidade e uso do } \\
\text { conhecimento que utiliza tecnologias e estruturas organizacionais para } \\
\text { sua realização. }\end{array}$ \\
\hline $\begin{array}{l}\text { JENNEX, SMOLNIK E E consiste em capturar o conhecimento certo, disponibilizando para o } \\
\text { CROASDELL (2009) }\end{array}$ & $\begin{array}{l}\text { GC } \\
\text { usuário certo, e utilizando esse conhecimento para melhorar o } \\
\text { desempenho individual ou organizacional. }\end{array}$ \\
\hline $\begin{array}{l}\text { NORTH E KUMTA } \\
\text { (2014) }\end{array}$ & $\begin{array}{l}\text { GC habilita os indivíduos, grupos, organizações, redes e nações a criar, } \\
\text { compartilhar e aplicar conhecimento de forma sistemática, para atingir } \\
\text { seus objetivos estratégicos e operacionais. GC incrementa a eficiência e } \\
\text { efetividade das operações por meio da melhoria da qualidade competitiva } \\
\text { (inovação) e do desenvolvimento de uma organização que aprende. }\end{array}$ \\
\hline
\end{tabular}

Fonte: Adaptado de Alarcon (2015); Dávila (2016)

Para facilitar ainda mais o entendimento sobre a gestão do conhecimento é necessária à distinção de três elementos: dados, que são obtidos por máquinas, é quantificáveis e de fácil transferência; as informações, que são dados atribuídos de relevância e propósito, requerem unidades de análise, há consenso em relação ao seu significado e normalmente exige mediação humana; e o conhecimento, informação da mente humana, que inclui reflexão, síntese e contexto, é de difícil estruturação e mensuração por máquinas (DAVENPORT; PRUSAK, 1998 apud ALARCON 2015).

$\mathrm{Na}$ percepção de Waltz (2003) as dimensões da gestão do conhecimento envolvem três componentes fundamentais: as pessoas, que devem lidar com a cultura organizacional e com as estruturas da organização, com o intuito de criar uma aprendizagem colaborativa; os processos, que devem também fomentar um ambiente para troca, descoberta, retenção, uso e reuso do conhecimento; e as tecnologias que devem ser aplicadas para qualificar os processos e a capacidade intelectual das pessoas.

Seguinte este pensamento Servin (2005) complementa esses três componentes, nas quais: as pessoas, dotadas de uma história de vida, possuem valores e comportamento, que interagem com a cultura organizacional e por ela são afetados; os processos, onde as organizações são estruturadas de forma a abrigar processos inerentes a sua gestão; e a tecnologia que é o meio para conectar as pessoas ao processo organizacional, atuando como ligação e contribuindo com a gestão do conhecimento.

Partindo de que as organizações são compostas por pessoas, processo e tecnologias, Brito (2008) diz que todas por mais distintas que sejam possuem um conhecimento organizacional, e é por este que de forma coletiva as pessoas conseguem executar tarefas que não conseguiriam fazê-la de 
forma isolada. Essas tarefas criam valor aos processos e aos envolvidos com a organização, e assim é traduzido nos produtos e serviços que ela oferece. Desta forma a gestão do conhecimento é uma forma específica de fazer com que o conhecimento produzido na empresa, seja mantido na própria organização, independente de quem o produziu.

A gestão do conhecimento é responsável pelas transformações que qualificam a empresa na busca por competitividade, pois auxilia no processo de geração de inovações e organiza as informações para os possíveis avanços de pesquisa e desenvolvimento, sendo assim a gestão do conhecimento pode ser um método eficaz para que as inovações comecem a emergir no ambiente organizacional (PICININ; KOVALESKI; RAIMUNDI, 2010).

Segundo Nonaka e Takeuchi, (1997) quando as organizações inovam, elas além de processarem informações de fora para dentro a fim de solucionar os problemas existentes, elas criam novos conhecimento e informações, de dentro para fora, e com isto redefinem tanto os problemas quanto as soluções, e assim recriar seu meio organizacional.

O Manual de Oslo que busca orientar diretrizes para coletas e interpretações de dados sobre inovação aponta quatro abordagens da inovação: inovação no produto que trata de mudanças significativas nas potencialidades de produtos e serviços; inovações de processo que tratam de mudanças significativas nos métodos de produção e distribuição; inovações de marketing, que tratam da implementação de novos métodos em design, embalagem, promoção ou preços; e inovação organizacional que trata das mudanças nas práticas de negócios, no local de trabalho e nas relações externas da empresa (OCDE, 2006).

Sendo assim a gestão da inovação é um processo estruturado, contínuo e facilitador para que as organizações consigam gerá-la e criar mais valor as inovações. É um processo baseado no conhecimento que pode ser modelado em diversos estágios. Dessa forma a gestão da inovação em uma empresa, frequentemente inclui a gestão da propriedade intelectual, das oportunidades tecnológicas, dos recursos para inovação, da transferência de tecnologia e das empresas nascentes na própria empresa (GRIZENDI, 2011).

Kaplan e Norton (2004) mencionam que a gestão da inovação inclui quatro importantes etapas. A primeira é identificar oportunidades de novos produtos e serviços; seguida por gerenciar o portfólio de pesquisa e desenvolvimento; projetar e desenvolver novos produtos e serviços; e lançar novos produtos e serviços no mercado.

\section{MÉTODO DE ESTUDO}

Nesta seção descreve-se o tipo do estudo, definição da amostra, modelo conceitual para análise bibliométrica e os procedimentos para a coleta dos dados.

\subsection{Tipo de Estudo}

O presente estudo foi desenvolvido a partir de uma pesquisa bibliométrica (Rostaing, 1997; Silva, 2004; Macedo, Casa Nova \& Almeida, 2009) de cunho quantitativo (MALHOTRA, 2006; HAIR et 
al, 2005), objetivando ampliar o conhecimento referente às publicações relacionadas à gestão do conhecimento (knowledge management) e a gestão da inovação (innovation management), na base de dados Web of Science, no período de 1945 a 2016. Além dos estudos apresentados que dão suporte para o estudo, cabe salientar que existem outros estudos na literatura que destacam-se na área da Administração. Cita-se o estudo de Paulista, Campos e Turrioni (2010) que identificou os artigos mais citados e os principais autores sobre gestão do conhecimento no período de (19952010).

Lei de Lotka (LL), que é um princípio que estuda sobre a produtividade dos autores de um determinado campo científico. Esta Lei da produtividade parte da premissa de que, proporcionalmente, poucos autores publicam mais (e são mais citados) do que muitos autores, que publicam menos. Esta distribuição é verificada em campos avançados da ciência. Moretti e Campanário apud Alvarado (2002) salientam que esta lei tem a seguinte definição: Para estabelecer a parte com que homens de diferentes qualidades contribuem ao progresso da ciência, Lotka (1926) estabeleceu os fundamentos da lei do quadrado inverso, afirmando que o número de autores que fazem $n$ contribuições em um determinado campo científico é aproximadamente $1 / n^{2}$ daqueles que fazem uma só contribuição. A proporção daqueles que fazem uma única contribuição é de mais ou menos $60 \%$.

\subsection{Definição da Amostra}

Os dados para realização desta pesquisa foram coletados por meio da base Web of Science do Institute for Scientific Information (ISI). Segundo Franceschet (2010) o ISI foi fundado por Eugene Garfield em 1960 e adquirida pela Thomson (hoje Thompson-Reuters) em 1992, e consiste em uma das maiores companhias do mundo da informação.

A Web of Science é uma base que indexa somente os periódicos mais citados em suas áreas de conhecimento. É ainda um índice de citações na web, que além de identificar as citações recebidas, as referências utilizadas, e outros registros, pode-se analisar a produção científica através do cálculo de índices bibliométricos e o percentual de autocitações, bem como a elaboração de rankings de vários parâmetros (CAPES 2011).

As referências de todos os itens indexados são extraídas e a interface das referências citadas lista todas as citações de trabalhos às obras de um autor, independentemente dos itens citados serem indexados pela Web of Science ou não (Bar-llan, 2008). Para tanto, a partir do mecanismo de busca da Web of Science, utilizando como palavras-chaves, Knowledge management e innovation management delimitando a busca para o período de 1945 a 2016 (71 anos) foram buscadas as publicações para análise.

\subsection{Modelo conceitual para Análise Bibliométrica} Quadro 2.

O modelo conceitual que norteia a análise bibliométrica, segue os indicadores expostos no 
Quadro 2 - Modelo conceitual para análise bibliométrica

\begin{tabular}{|l|l|}
\hline \multicolumn{2}{|c|}{ Características gerais das publicações } \\
\hline Total de publicações & Instituições \\
\hline Áreas temáticas & Agências financiadoras \\
\hline Tipos de documentos & Países \\
\hline Ano das publicações & \\
\hline Autores & \\
\hline Título das fontes & \\
\hline Fonte: Elaborado pelos autores (2016)
\end{tabular}

Conforme se apresenta no Quadro 2, as características serão apresentadas na sequência, com base nos resultados expressos pela pesquisa.

\subsection{Procedimentos para Coleta dos Dados}

Para a realização da pesquisa inicialmente digitou-se as palavras knowledge management $e$ innovation management como tópico no campo de pesquisa da Web of Science, delimitando-se o período de 1945 a 2016 (71 anos). Dessa forma, foram levantadas as informações: número total de publicações, áreas temáticas, tipo de documentos, ano das publicações, autores, título das fontes, instituições, países e idiomas.

\section{CARACTERÍSTICAS GERAIS DAS PUBLICAÇÕES SOBRE GESTÃO DO CONHECIMENTO E GESTÃO DA INOVAÇÃO NA WEB OF SCIENCE}

Na pesquisa realizada com os tópicos gestão do conhecimento e gestão da inovação foram encontradas 372 publicações. A seguir estão apresentadas as características gerais das publicações relacionadas ao tema de acordo com as seguintes categorias: áreas temáticas, tipo de documentos, ano das publicações, autores, título das fontes, instituições, países e idiomas.

\section{1 Áreas Temáticas}

No Quadro 3 apresenta às dez principais áreas temáticas relacionadas ao tema de acordo com o número de publicações. As 10 áreas selecionadas apresentam evidências do crescimento das áreas temáticas relacionadas sobre o tema.

Quadro 3 - Áreas temáticas no estudo sobre gestão do conhecimento e gestão da inovação

\begin{tabular}{|l|c|l|c|}
\hline \multicolumn{1}{|c|}{ Área temática } & $\begin{array}{c}\text { № de } \\
\text { publicações }\end{array}$ & \multicolumn{1}{|c|}{ Área temática } & $\begin{array}{c}\text { № de } \\
\text { publicações }\end{array}$ \\
\hline $\begin{array}{l}1^{\circ} \text { Business economics } \\
\text { (Economia empresarial) }\end{array}$ & 240 & $\begin{array}{l}6^{\circ} \text { Social science other topics } \\
\text { (Ciências sociais) }\end{array}$ & 19 \\
\hline
\end{tabular}

Estudos do CEPE [ISSN 1982-6729]. Santa Cruz do Sul, n. 44, p. 113-126, jul./dez. 2016. https://online.unisc.br/seer/index.php/cepe/index 


\begin{tabular}{|l|c|l|c|}
\hline $\begin{array}{l}2^{\circ} \text { Operation research } \\
\text { management science (Gestão } \\
\text { de operações) }\end{array}$ & 88 & $\begin{array}{l}7^{\circ} \text { Public administration } \\
\text { (Administração pública) }\end{array}$ & 18 \\
\hline $3^{\circ}$ Engineering (Engenharia) & 87 & $\begin{array}{l}8^{\circ} \text { Education educational } \\
\text { research (Pesquisas } \\
\text { educacionais) }\end{array}$ & 17 \\
\hline $\begin{array}{l}4^{\circ} \text { Computer science (Ciência } \\
\text { da computação) }\end{array}$ & 86 & $9^{\circ}$ Psychology (Psicologia) & 11 \\
\hline $\begin{array}{l}5^{\circ} \text { Information science library } \\
\text { science (Ciência da } \\
\text { informação/biblioteconomia) }\end{array}$ & 50 & $\begin{array}{l}10^{\circ} \text { Telecommunications } \\
\text { (Telecomunicação) }\end{array}$ & \\
\hline
\end{tabular}

Fonte: Web of Science (2016)

A principal área temática relativa às publicações sobre a temática da gestão do conhecimento (Knowledge management) e da gestão da inovação (innovation management) foi a Economia Empresarial (Business Economics). Com menor expressão destacam-se os temas Pesquisas educacionais (Education educational research), Psicologia (Psychology) e Telecomunicação (Telecomunications).

\subsection{Tipos de Documentos}

No Quadro 4 apresenta os tipos de documentos referentes às publicações encontradas.

Quadro 4 - Classificação das publicações quanto ao tipo

\begin{tabular}{|l|c|c|}
\hline \multicolumn{1}{|c|}{ Tipos de publicação } & Frequência & Percentual \\
\hline Proceedings paper & 248 & $66,6 \%$ \\
\hline Article & 104 & $27,9 \%$ \\
\hline Book review & 9 & $2,4 \%$ \\
\hline Editorial material & 8 & $2,1 \%$ \\
\hline Review & 7 & $1,8 \%$ \\
\hline Meeting abstract & 4 & $1,5 \%$ \\
\hline
\end{tabular}

Fonte: Web Of Science (2016)

A maioria das publicações encontradas são proceeding papers, artigos, revisão, evidenciando o caráter científico das mesmas.

\subsection{Publicações por Ano}

A Figura 1 apresenta a quantidade de artigos publicados por ano relacionado ao tema, sob a ótica evolutiva.

Estudos do CEPE [ISSN 1982-6729]. Santa Cruz do Sul, n. 44, p. 113-126, jul./dez. 2016. 
Figura 1 - Publicações por ano

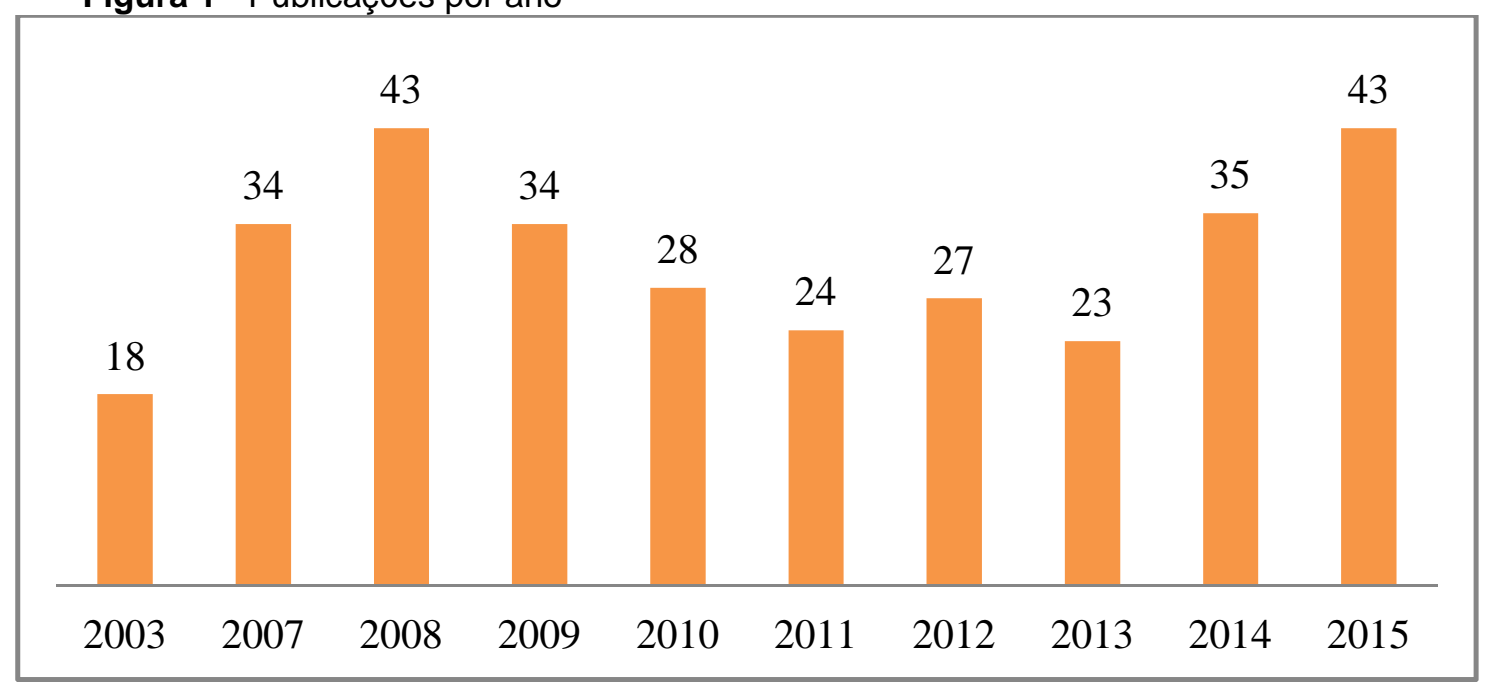

Fonte: Web of Science (2016)

No período compreendido entre 1945 e 2016, constatou-se que o número de publicações aumentou gradativamente até o ano de 2008, após teve ápices e declives até o ano de 2015, onde equiparou o ano de 2008.

\subsection{Principais Autores}

No Quadro 5 apresenta os 8 autores que mais publicaram no período analisado.

Quadro 5 - Quantidade de artigos publicados por autor

\begin{tabular}{|l|c|l|c|}
\hline \multicolumn{1}{|c|}{ Autores } & Artigos publicados & \multicolumn{1}{c|}{ Autores } & Artigos publicados \\
\hline An $x m$ & 3 & Gloet $m$ & 3 \\
\hline Corso $m$ & 3 & Hassan $s$ & 3 \\
\hline Deng HP & 3 & Liy & 3 \\
\hline Donate $m j$ & 3 & Samson D & 3 \\
\hline Fonte: Web Of Science $(2016)$ & &
\end{tabular}

Fonte: Web Of Science (2016)

Os principais autores são de inúmeras instituições de ensino e destacam-se nas diversas áreas de gestão, com transversalidade em áreas de sistemas de informação, engenharia de sistemas, gestão de operações, etc. Shahizan Hassan, atualmente é professor de sistemas de informação empresariais na Universidade Utara Malásia. Danny Samson é desde 1988 professor de gestão de operações e gestão de marketing do departamento de administração da Universidade de Melbourne. Mario Javier Donate é professor da Universidade de Castilha-La Mancha na Espanha e Dr. Mariano Corso é professor da Faculdade de Engenharia de Sistemas do Politécnico de Milão. 


\subsection{Títulos das Fontes}

No Quadro 6 apresenta as principais fontes de publicações relacionadas ao tema.

Quadro 6 - Principais fontes

\begin{tabular}{|l|c|l|c|}
\hline \multicolumn{1}{|c|}{ Título da fonte } & $\begin{array}{c}\text { No de } \\
\text { artigos }\end{array}$ & \multicolumn{1}{|c|}{ Título da fonte } & $\begin{array}{c}\text { No de } \\
\text { artigos }\end{array}$ \\
\hline $\begin{array}{l}\text { International journal of technology } \\
\text { management }\end{array}$ & 15 & Baltic journal of management & 5 \\
\hline $\begin{array}{l}\text { Proceedings of the European } \\
\text { conference on knowledge } \\
\text { management }\end{array}$ & 12 & $\begin{array}{l}\text { International conference on industrial } \\
\text { engineering and engineering } \\
\text { management ieem }\end{array}$ & 5 \\
\hline $\begin{array}{l}\text { Journal of Knowledge } \\
\text { management }\end{array}$ & 10 & $\begin{array}{l}\text { International journal of information } \\
\text { management }\end{array}$ & 5 \\
\hline $\begin{array}{l}\text { International conference on } \\
\text { wireless communications } \\
\text { networking and mobile computing }\end{array}$ & 6 & $\begin{array}{l}\text { Proceedings of the 13th European } \\
\text { conference on knowledge } \\
\text { management vols 1 and 2 }\end{array}$ & 5 \\
\hline $\begin{array}{l}\text { Ismot 07 Proceedings of the fifth } \\
\text { international symposium on } \\
\text { management on technology vols } \\
1 \text { and } 2\end{array}$ & 6 & $\begin{array}{l}\text { Proceedings of the 14th European } \\
\text { conference on knowledge } \\
\text { management eckm 2013 vols 1 and } \\
2\end{array}$ & 5 \\
\hline
\end{tabular}

Fonte: Web Of Science (2016)

A maioria das publicações referente ao tema foi publicada nos periódicos International journal of technology management, Proceedings of the European conference on knowledge management, Journal of Knowledge management, International conference on wireless communications networking and mobile computing e Ismot 07 Proceedings of the fifth international symposium on management on technology vols 1 and 2.

\subsection{Principais Instituições}

No Quadro 7 apresenta as instituições que mais publicaram trabalhos relacionados ao tema.

Quadro 7 - Principais Instituições

\begin{tabular}{|l|c|}
\hline \multicolumn{1}{|c|}{ Instituição } & № de Artigos \\
\hline Wuhan University of Technology & 9 \\
\hline South China University of Technology & 7 \\
\hline Zhejiang University & 7 \\
\hline University of Murcia & 6 \\
\hline Tianjin Polytechnic University & 3 \\
\hline Universidade de São Paulo & 3 \\
\hline
\end{tabular}

Estudos do CEPE [ISSN 1982-6729]. Santa Cruz do Sul, n. 44, p. 113-126, jul./dez. 2016. https://online.unisc.br/seer/index.php/cepe/index 


\begin{tabular}{|l|c|}
\hline University Utara Malaysia & 3 \\
\hline University of Manchester & 3 \\
\hline University of Melbourne & 3 \\
\hline University of Science Technology of China & 3 \\
\hline
\end{tabular}

Fonte: Web of Science (2016)

As instituições que mais se destacam nas publicações relacionadas à gestão do conhecimento e gestão da inovação são: Wuhan University of Technology, South China University of Technology, Zhejiang University, University of Murcia, Tianjin Polytechnic University, Universidade de São Paulo, University Utara Malaysia, University of Manchester, University of Melbourne e University of Science Technology of China. Assim, verifica-se que metade das instituições de concentram na China, sendo as demais da Espanha, Malásia, Inglaterra e Brasil.

\subsection{Principais Países e Idiomas}

No Quadro 8 apresenta os principais países que mais publicaram trabalhos relacionados ao tema.

\begin{tabular}{|c|c|c|c|}
\hline $\begin{array}{l}\text { Países } \\
\end{array}$ & № de artigos & Países & № de artigos \\
\hline $1^{\circ}$ China & 123 & 6ํMalásia & 14 \\
\hline 2ํㅗastos Unidos da América & 27 & 7º Austrália & 13 \\
\hline 3ํㅗspanha & 25 & 8 Alemanha & 8 \\
\hline $4^{\circ}$ Taiwan & 25 & 9ㅇ Itália & 7 \\
\hline $5^{\circ}$ Inglaterra & 22 & $10^{\circ}$ Portugal & 7 \\
\hline
\end{tabular}

Quanto ao número de publicações por países, a China lidera o ranking de publicações, seguido pelos Estados Unidos, Espanha, Taiwan e Inglaterra. Dessa forma, pode-se inferir que nesses países se encontram a maior parte das instituições que possuem pesquisas relacionadas à temática. Os idiomas se dividem em dois English (361) e Chinese (5).

\section{CONSIDERAÇÕES FINAIS}

A Gestão do Conhecimento é um processo para criação, captura, armazenamento, disseminação, uso e proteção do conhecimento importante para a empresa. A Gestão do Conhecimento, por meio de suas práticas, objetiva organizar de forma estratégica os conhecimentos dos colaboradores e os conhecimentos externos, que são fundamentais para o sucesso do negócio. A Gestão da Inovação envolve o processo de desenvolvimento tecnológico, a aplicação da tecnologia 
em produtos ou processos e a adoção e difusão tecnológica na empresa. Ambos os conceitos são fundamentais para a gestão das organizações, e são necessárias a atuação conjunta, bem como os processos se relacionam. Não á inovação sem gestão do conhecimento, ou vice versa.

Segundo Drucker (2003) não seremos limitados pela informação que temos. Seremos limitados por nossa habilidade de processar esta informação. Atualmente a GC e GI são temas que estão cada vez mais difundidos e facilmente podemos encontrar práticas em todas as empresas e até mesmo no seu ambiente pessoal, tudo isso em função do avanço da tecnologia da informação. E, essas práticas que complementam a gestão dos processos importantes que qualquer empresa ou organização precisam conhecer e estruturar, mas não são as únicas. À medida que o mercado exige e as empresas adquirem conhecimento e experiência, é natural que surjam estratégias novas baseadas em cada situação, baseadas em cada inovação.

O estudo das publicações é de suma importância para entender a evolução da temática, a evolução dos conceitos, as pesquisas que estão sendo realizados, quais os avanços e como podemos avançar em novas pesquisas.

A análise das publicações sobre Knowledge management (Gestão do conhecimento) and innovation management (Gestão da inovação) na base de dados Web of Science, evidenciou 372 publicações relacionadas ao tema, estando relacionadas principalmente às áreas temáticas: Business economics (Economia Empresarial), Operations research management science (Gestão de Operações), Engineering (Engenharias), Computer science (Ciência da Computação), Information science library science (Ciência da informação/biblioteconomia), Social science other topics (Ciências Sociais), Public administration (Administração Pública), Education educational research (Pesquisas Educacionais), Psychology (Psicologia), Telecomunications (Telecomunicações). Constatou-se que a maioria das publicações encontradas são papers (66\%) e artigos (27\%), constatou um crescimento com sobre a produção científica com oscilações nos últimos anos, tento ápices nos anos de 2008 e 2015 .

A maioria das publicações referentes ao tema foi publicada nos periódicos: International journal of technology management, Proceedings of the European conference on knowledge management, Journal of Knowledge management, International conference on wireless communications networking and mobile computing e Ismot 07 Proceedings of the fifth international symposium on management on technology vols 1 and 2. Também constatou-se que a China lidera o ranking de publicações, seguidos pelos Estados Unidos e Espanha, e que o idioma inglês é o predominante nas publicações.

No decorrer do trabalho, foi possível verificar a utilidade de mecanismos de busca como a web of Science para a realização de pesquisas acadêmicas, que servem de ferramenta para que a comunidade acadêmica tenha acesso às publicações bem como busque informações a respeito da evolução de seus temas de interesse.

Este estudo apresenta a evolução dos temas, os avanços que possibilitam ao leitor a compreensão e a difusão de novos conhecimentos. Para estudos futuros, percebe-se a importância da investigação de trabalhos e avanços científicos por meio de um estudo documental e uma investigação com experts sobre na temática. 


\section{REFERÊNCIAS}

ALVARADO, R. U. A Lei de Lotka na bibliometria brasileira. Ciência da Informação, v. 31, n. 2, oct. 2002. ISSN 1518-8353. Disponível em: http://revista.ibict.br/ciinf/article/view/956. Acesso em: 05 de agosto de 2016. DOI: http://dx.doi.org/10.18225/ci.inf..v31i2.956.

ALARCON, D, F. Diretrizes Para Práticas de Gestão do Conhecimento na Educação a Distância. Florianópolis. 213 f. Tese (Doutorado em Engenharia e Gestão do Conhecimento). Universidade Federal de Santa Catarina, 2015.

ALAVI, M.; LEIDNER, D, E. Review: Knowledge management and knowledge management systems: Conceptual foundations and research issues. MIS quarterly, no 25, p. 107-136, 2001.

BAR-ILAN J. Which h-index? A comparison of wos, scopus and google scholar. Scientometrics, v. 74, n. 2, pp. 257-271, 2008.

BERH, R, R.; NASCIMENTO, S, P. A gestão do conhecimento como técnica de controle: uma abordagem crítica da conversão do conhecimento tácito em explícito. CADERNOS EBAPE. BR, v. 6, oㅜ 5, p. 1-11, Março, 2008.

BRITO, L, M, P. Gestão do conhecimento - instrumento de apropriação pelo capital do saber do trabalhador. Cadernos de Educação/FaE/PPGE/UFPel. Pelotas [30]: p. 135-148, janeiro/junho, 2008.

COORDENAÇÃO DE APERFEIÇOAMENTO DE PESSOAL DE NÍVEL SUPERIOR. Sobre Web of Science. Recuperado de: <http://buscador.periodicos.capes.gov.br.ez47.periodicos.

Capes.gov.br/V/DNA9QG6PQIPNQSNNEG55MSCFIKMIU8IL8DKSBPYEP1XU8H49XB-

34072?Func=find-db-info\&doc_num=000002653>. Acesso em: 20 Julho de 2012.

DAVENPORT, T. H.; PRUSAK, L. Conhecimento empresarial. Rio de Janeiro: Campus, 1998.

DRUCKER, P. Inovação e espírito empreendedor. São Paulo: $7^{\text {ạ }}$ ed., Pioneira, 2003.

FRANCESCHET, M. A comparison of bibliometric indicators for computer science scholars and journals on web of science and google scholar. Scientometrics, v. 83, n. 1, p 243-258, 2010.

GIUOMAR, T, F, M. Gestão do Conhecimento: A importância da Inovação e da Competitividade numa Organização do Século XXI. Coimbra, 150 f. Dissertação (Mestrado em Informação, Comunicação e Novos Média) Universidade de Coimbra, 2014.

GURTEEN, D. Knowledge, creativity and innovation. Journal of knowledge Management, v. 2, n. 1, p. $5-13,1998$.

GRIZENDI, E. Manual de orientações gerais sobre inovação. (FINEP, 2011). Disponível em: http://www.investexportbrasil.gov.br/sites/default/files/publicacoes/estudos/PUBEstudosManualDalnov acao.pdf. Acesso em: 15 de agosto de 2016.

HAIR Jr., J. F.; ANDERSON, R. E.; TATHAM, R. L.; BL ACK, W. C. Análise Multivariada de Dados. 5.ed. Porto Alegre: Bookman, 2005.

JENNEX, M. E.; SMOLNIK, S.; CROASDELL, D. T. Towards a consensus knowledge management success definition. VINE, v. 39, n. 2, p. 174-188, 2009.

KAPLAN, R. S.; NORTON, D. P. Kaplan e Norton na prática. Rio de Janeiro: Campus, 2004.

MACEDO, M. A. S.; NOVA, S. P. C. C.; ALMEIDA, K. Mapeamento e análise bibliométrica da utilização da Análise Envoltória de Dados (DEA) em estudos em contabilidade e administração.

Contabilidade, Gestão e Governança, v. 12, n. 3, p. 87-101, 2009. 
MALHOTRA, N. K. Pesquisa de Marketing: uma orientação aplicada. 4. ed. Porto Alegre: Bookman,2006.

MANUAL DE OSLO: Diretrizes para coleta e interpretação de dados sobre inovação. (OCDE FINEP, 2006) Disponível em: http://www.finep.gov.br/images/apoio-e-financiamento/manualoslo.pdf. Acesso em 15 de agosto de 2016.

MORETTI, S. L. A.; CAMPANÁRIO, M. A. A produção intelectual brasileira em responsabilidade social empresarial - rse sob a ótica da bibliometria. Revista de administração contemporânea, v. 13, n. n. 8 , p. 68-86, 2009.

NONAKA, I; TAKEUCHI, H. Criação de Conhecimento na Empresa: como as empresas japonesas geram a dinâmica da inovação. Rio de Janeiro: Campus, 1997.

NORTH, K.; KUMTA, G. Knowledge management: Value creation through organizational learning. Switzerland: Springer Science \& Business Media, 2014.

PICININ, C. T.; KOVALESKI, U. L.; RAIMUNDI, C. V. Gestão do conhecimento e inovação: um enfoque logístico. Revista Eletrônica FAFIT/FACIC, v. 1, n. 1, p. 17-29, Jan/Jun, 2009.

PAULISTA, P, H.; CAMPOS, D, F.; TURRIONI, J, B. ANÁLISE BIBLIOMÉTRICA DA GESTÃO DO CONHECIMENTO . In: XXX ENCONTRO NACIONAL DE ENGENHARIA DE PRODUÇÃO, São Carlos, SP, 2010. Anais do Enegep, 2010.

ROSTAING, H. La bibliométrie et ses techniques. Toulouse: Sciences de la Société; Marseille: Centre de Recherche Rétrospective de Marseille, 1997.

ROSÁRIO, I, A, A. Gestão da inovação numa empresa de serviços de engenharia civil. Porto, 127 f. Dissertação (Mestrado em Engenharia Civil) Universidade do Porto, 2011.

SERVIN, G. ABC of Knowledge Management. NHS National Library for Health. 2005. Disponível em: http://www.fao.org/fileadmin/user_upload/knowledge/docs/ABC_of_KM.pdf. Acesso em: 25 de setembro de 2016.

SILVA, M. R. Análise bibliométrica da produção científica docente do programa de pós-graduação em educação especial/UFSCar. São Carlos. Dissertação (Mestrado em Educação Especial) Universidade Federal de São Carlos, São Carlos, 2004.

SVEIBY, K. E. A nova riqueza das organizações: gerenciando e avaliando patrimônio de conhecimento. Rio de janeiro: campus, 1998.

TERRA, J. C. C. Gestão do conhecimento: o grande desafio empresarial. 2005.

WALTZ, E. Knowledge management in the intelligence enterprise. Artech House information warfare library, 2003. 